Bio - grafía. Escritos sobre la Biología y su Enseñanza. ISSN 2027-1034

Edición Extraordinaria. p.p. 966 - 974

Memorias del IX Encuentro Nacional de Experiencias en Enseñanza de la Biología y la Educación Ambiental. IV Congreso Nacional de Investigación en Enseñanza de la Biología.

\title{
ENSEÑANZA-APRENDIZAJE DEL ORDEN TRICÓPTERA EN GRADO SEXTO: ANÁLISIS MEDIANTE UNAHIPÓTESIS DE PROGRESIÓN
}

\section{TEACHING-UNDERST ANDING OF THE TRICÓPTERA ORDER IN SIXTH GRADE: AN ALYSIS THROUGH A HYPOTHESIS OF PROGRESSION}

\author{
Jairo Alonso Forero Anaya \\ Robinson Roa Acosta
}

\section{RESUMEN}

Esta investigación hizo seguimiento al aprendizaje de estudiantes de grado sexto a través de una hipótesis de progresión. Se desarrolló un itinerario didáctico en el que se abordaron temáticas del Orden Tricóptera con 12 estudiantes de grado sexto de un colegio público de Bogotá.

Para tal objetivo se construyeron actividades escolares que cubrieron un periodo de dos meses con temáticas específicas de ciencias naturales (hábitat, adaptación morfológica, relaciones ecológicas, hábitos tróficos y acción antrópica)

Los datos obtenidos fueron tratados a través de un enfoque cualitativo de forma descriptiva e interpretativa.

La hipótesis de progresión permitió hacer seguimiento al aprendizaje de los estudiantes sobre los Tricópteros, desde un conocimiento simple hacia uno complejo. Por lo tanto, la concordancia entre la secuenciación de los contenidos y las actividades desarrolladas fue pertinente para esta investigación.

\section{PALABRAS CLAVE}

Enseñanza, aprendizaje, Tricóptera, hipótesis de progresión.

\section{ABSTRACT}

This research followed the learning of sixth grade students through a hypothesis of progression. A didactic itinerary was developed in it was tackle topics of the Tricóptera order with twelve students of sixth grade of a public school of Bogotá.

For such an aim was construct scholar activities that covered a period of two months half past school with specifics thematics of natural sciences (habitat, morphological adaptation, ecological relationships, trophies habits and anthropic action). 
Bio - grafía. Escritos sobre la Biología y su Enseñanza. ISSN 2027-1034

Edición Extraordinaria. p.p. 966 - 974

Memorias del IX Encuentro Nacional de Experiencias en Enseñanza de la Biología y la Educación Ambiental. IV Congreso Nacional de Investigación en Enseñanza de la Biología.

The data obtained were treated through a quantitative approach of descriptive and interpretative way.

The progression hypothesis made it possible to follow up the students' learning about the Tricópteros, who went from simple knowledge to complex knowledge in the measure that their previous ideas were confronted with new ones.

The concordance between the sequencing of the contents and the activities developed to follow the student's learning was relevant this research.

\section{KEY WORDS}

Learning, teaching, Tricóptera, hypothesis of progression.

\section{INTRODUCCIÓN}

Los Tricópteros son insectos que en su estado larvario dan cuenta de condiciones específicas del ambiente. No solo son bioindicadores de la salud de los ecosistemas lóticos, sino que además son parte fundamental en el movimiento de nutrientes a lo largo de las redes tróficas. En Colombia, hay un conocimiento amplio acerca de la importancia de este Orden debido a estudios realizados con la anatomía, ecología, cultivos en cautiverio y la determinación de la salud de ecosistemas de la región andina a través de la presencia de estas poblaciones.

Muchas de las especies, que hacen parte del inventario biológico de nuestro país pasan desapercibidas porque no se da a conocer lo que representan para los ecosistemas. Gran parte del conocimiento acerca de los Tricópteros está limitado a artículos especializados en el tema.

En tal sentido, es importante que el conocimiento de la biodiversidad pueda ser llevado a la escuela a través de procesos de enseñanza-aprendizaje con el fin de que los estudiantes valoren este patrimonio. Esta investigación que correspondió a un trabajo de tesis de maestría tuvo como objetivos construir una hipótesis de progresión sobre del Orden Tricóptera para hacer seguimiento al aprendizaje de estudiantes de grado sexto a través de actividades escolares de ciencias naturales.

\section{METODOLOGÍA DE LA INVESTIGACIÓN}

En esta investigación participaron 12 estudiantes de grado sexto (entre los 12 y 13 años) de un colegio público de Bogotá, y un profesor de biología que dirigió la enseñanza del orden Tricóptera durante dos meses y medio.

La investigación se organizó en tres fases: 
Bio - grafía. Escritos sobre la Biología y su Enseñanza. ISSN 2027-1034

Edición Extraordinaria. p.p. 966 - 974

Memorias del IX Encuentro Nacional de Experiencias en Enseñanza de la Biología y la Educación Ambiental. IV Congreso Nacional de Investigación en Enseñanza de la Biología.

Primera fase: de las correspondientes consultas bibliográficas se tuvieron en cuenta tres referentes teóricos (Conocimiento profesional del profesor de ciencias, perspectivas de investigación en la escuela y diversidad biológica en Colombia).

Segunda fase: consistió en la construcción de las hipótesis de progresión teniendo en cuenta las propuestas de Cañal y Porlán (1987); García y Rivero (1993); García (1994); Martin (1994); Ángel y De Longhi (2006) y Valbuena (2007) para cada una de las temáticas sobre el Orden Tricóptera:

- Nivel 1: Las ideas de los estudiantes acerca de los Tricópteros se muestran lineales y sin causalidad. No se establecen relaciones entre los elementos constitutivos del ambiente de estas poblaciones. Predomina una visión inmediata, poco relacional y carente de complejidad.

- Nivel 2: Tras la instrucción, los estudiantes comienzan a mostrar ideas en las que se establecen relaciones sencillas entre las poblaciones de Tricópteros y los componentes constitutivos de su ambiente. La aparición de lo poco evidente es parte de este nivel de desarrollo además de la utilización de palabras o términos más estructurados.

- Nivel 3: Se establecen relaciones claras de causalidad entre las poblaciones de Tricópteros y cada uno de los elementos constitutivos de su ambiente. Las relaciones son multicausales y en forma de redes de relación que muestran un grado propio de avance para el conocimiento escolar deseable en los estudiantes.

Los instrumentos utilizados para la recolección de datos fueron:

- Diario de campo del profesor.

- Producciones escritas de los estudiantes acerca del impacto del hombre sobre los ecosistemas acuáticos y en específico con los Tricópteros y las relaciones biológicas, ecológicas y sociales que afectan la dinámica de sus hábitats.

- Guías de trabajo que desarrollaron los estudiantes.

Tercera fase: consistió en el análisis de los datos obtenidos (Correa, Cubero y García, 1994; García, 1994 y 1995; Porlán, 1987, 1993 y 1998), se realizó una descripción cualitativa y cuantitativa con el fin de estimar el aprendizaje de los estudiantes.

La tabla muestra las actividades que hicieron parte del itinerario didáctico: 
Bio - grafía. Escritos sobre la Biología y su Enseñanza. ISSN 2027-1034

Edición Extraordinaria. p.p. 966 - 974

Memorias del IX Encuentro Nacional de Experiencias en Enseñanza de la Biología y la Educación Ambiental. IV Congreso Nacional de Investigación en Enseñanza de la Biología.

\begin{tabular}{|c|c|c|c|}
\hline & Temática & Metodología & $\begin{array}{l}\text { Conceptos } \\
\text { abordados }\end{array}$ \\
\hline 1 & Introducción a la biodiversidad biológica en Colombia & Inventario & $\begin{array}{l}\text { Hábitat, } \\
\text { antrópica }\end{array}$ \\
\hline 2 & Sistemas acuáticos colombianos & $\begin{array}{l}\text { Video charla } \\
\text { Dibujos } \\
\text { representativos }\end{array}$ & $\begin{array}{l}\text { Hábitat, Adaptación } \\
\text { morfológica, } \\
\text { Relaciones } \\
\text { ecológicas y Hábitos } \\
\text { tróficos }\end{array}$ \\
\hline 3 & Introducción a los Tricópteros & Guía ilustrada & $\begin{array}{l}\text { Hábitat, Adaptación } \\
\text { morfológica, } \\
\text { Relaciones } \\
\text { ecológicas y Hábitos } \\
\text { tróficos }\end{array}$ \\
\hline 4 & Análisis de especímenes & $\begin{array}{l}\text { Descripción de } \\
\text { estructuras }\end{array}$ & $\begin{array}{l}\text { Hábitat, Adaptación } \\
\text { morfológica, } \\
\text { Relaciones } \\
\text { ecológicas y Hábitos } \\
\text { tróficos }\end{array}$ \\
\hline 5 & El ambiente de los Tricópteros y sus adaptaciones. & Producción escrita & $\begin{array}{l}\text { Hábitat, Adaptación } \\
\text { morfológica, } \\
\text { Relaciones } \\
\text { ecológicas y Hábitos } \\
\text { tróficos }\end{array}$ \\
\hline 6 & Introducción a las relaciones ecológicas & $\begin{array}{l}\text { Video y diario de } \\
\text { clase }\end{array}$ & $\begin{array}{l}\text { Hábitat, Relaciones } \\
\text { ecológicas y Hábitos } \\
\text { tróficos }\end{array}$ \\
\hline 7 & Juego de simulación de redes tróficas & $\begin{array}{l}\text { Organización de } \\
\text { especímenes de } \\
\text { acuerdo a la } \\
\text { información } \\
\text { suministrada }\end{array}$ & $\begin{array}{l}\text { Adaptación } \\
\text { morfológica, } \\
\text { Relaciones } \\
\text { ecológicas y Hábitos } \\
\text { tróficos }\end{array}$ \\
\hline 8 & Ecosistemas acuáticos y su impacto antrópico & Debate por grupos & $\begin{array}{l}\text { Relaciones } \\
\text { ecológicas y Hábitos } \\
\text { tróficos }\end{array}$ \\
\hline
\end{tabular}


Bio - grafía. Escritos sobre la Biología y su Enseñanza. ISSN 2027-1034

Edición Extraordinaria. p.p. 966 - 974

Memorias del IX Encuentro Nacional de Experiencias en Enseñanza de la Biología y la

Educación Ambiental. IV Congreso Nacional de Investigación en Enseñanza de la Biología.

\begin{tabular}{|l|l|l|l|}
\hline 9 & Diagrama general de articulación de lo aprendido & Mapa conceptual & $\begin{array}{l}\text { Hábitat, Adaptación } \\
\text { morfológica, } \\
\text { Relaciones } \\
\text { ecológicas, Hábitos } \\
\text { tróficos y acción } \\
\text { antrópica }\end{array}$ \\
\hline
\end{tabular}

\section{RESULTADOS Y DISCUSIÓN}

A lo largo del desarrollo de las actividades del itinerario didáctico hubo una progresiva utilización de términos ecológicos, taxonómicos y morfológicos relacionados con los Tricópteros. Algunas evidencias de esto fueron las siguientes:

- Se mencionaron una gran cantidad de especies y de grupos taxonómicos que no aparecen en las actividades iniciales (actividad 1 y 2). Así por ejemplo, se citan los musgos, los hongos, otras especies de insectos y se mencionan las plantas como parte fundamental de los ecosistemas acuáticos.

- En algunos casos se presentó confusión a la hora de identificar los macroinvertebrados, pues en unos casos se les denomina por un término genérico (insectos,) y en otros por términos más específicos (Tricópteros).

- Al inicio del itinerario no apareció ninguna mención a los seres microscópicos que de igual forma son importantes en la dinámica de flujo de nutrientes en los ecosistemas. Resultados similares fueron encontrados por García (1994) en los que en las actividades iniciales, los estudiantes no señalaban nombres taxonómicos específicos para las especies constitutivas de un ecosistema acuático.

El problema del significado del término de microorganismos se integra en un problema más amplio que es el de la clasificación y denominación de los seres vivos. Por esta razón, al inicio del itinerario los estudiantes no identificaron los elementos del ecosistema con especies particulares, sino con agrupaciones de especies de forma general. Así, por ejemplo, en los inventarios que realizaron de los seres vivos representativos para los ecosistemas acuáticos colombianos, utilizan alusiones generalistas como "plantas", "peces", "aves" o "animales"

Con relación a componentes que hacen parte del hábitat de los Tricópteros los estudiantes coincidieron en afirmar que la construcción de las casas se realiza a partir de palos, hojarasca, piedrecillas, hojas y material vegetal. Además relacionaron la forma anatómica de los Tricópteros con los lugares específicos del rio que habitan, lo que permitió llegar hasta el nivel 2 de la hipótesis de progresión.

Con respecto a los coreotopos específicos, a partir de la forma de las estructuras anatómicas observadas en el laboratorio, los estudiantes establecieron las zonas del rio que estos individuos habitan. As í, los coreotopos de PCL (piedra corriente lenta) y (PRC) 
Bio - grafía. Escritos sobre la Biología y su Enseñanza. ISSN 2027-1034

Edición Extraordinaria. p.p. 966 - 974

Memorias del IX Encuentro Nacional de Experiencias en Enseñanza de la Biología y la Educación Ambiental. IV Congreso Nacional de Investigación en Enseñanza de la Biología.

piedra corriente rápida fueron los que aparecieron con mayor regularidad en las intervenciones de clase y en la práctica de laboratorio.

La forma causal predominate siguió siendo la casualidad mecánica y lineal que va asociada a la noción de cadena alimenticia. Al respecto, los estudiantes en las argumentaciones de clase comparten la afirmación, referida a los posibles intercambios de energía entre los depredadores y las presas. Sin embargo, en el debate, también hay estudiantes que se refieren a la interdependencia depredador-presa para la supervivencia de los Tricópteros. Así, la argumentación de las relaciones que se estaban estableciendo fueron sencillas pero se estaban teniendo en cuenta elementos del microcosmos que en las actividades anteriores no fueron mencionados.

\section{La hipótesis de progresión como instrumento de seguimiento del aprendizaje de los estudiantes}

Algunas de las evidencias del aprendizaje en los estudiantes a través del seguimiento de la hipótesis de progresión planteada fueron las siguientes:

- La utilización de un lenguaje de las ciencias naturales de acuerdo a las temáticas que se estaban abordando. Para los estudiantes palabras como Tricóptera, hábitat, relaciones ecológicas, hábitos tróficos, depredación, morfología, adaptación, refugios, y partes anatómicas como patas, agallas, uñas y mandíbulas se mencionaban como parte de la argumentación en las clases de ciencias.

- Las temáticas que mostraron una mayor cantidad de relaciones sistémicas fueron las de adaptación morfológica, hábitat, relaciones ecológicas y hábitos tróficos. Esto, porque a lo largo de las actividades propuestas se evidenció cómo se vinculaban aspectos morfológicos con la adaptación y con las formas en que los Tricópteros obtienen su alimento. Además, se relacionaron aspectos poco evidentes al inicio del itinerario como la luz solar, las algas y otros insectos, con la ecología de las poblaciones de Tricópteros.

- Finalmente, fue notorio en los estudiantes la forma en que compartieron las ideas aprendidas a lo largo del proceso, argumentando sus ideas acerca de los Tricópteros con los demás compañeros de clase.

Aunque se tuvieron en cuenta determinados temas a la hora de la realización de las actividades, emergieron en los estudiantes respuestas que permitieron evaluar otras temáticas que aún no se habían tratado. Un ejemplo de esto es que en la actividad número 3 en la que se pretendían abordar la temática de hábitat, los estudiantes mostraron avance en temas que se trataron anterior o después como relaciones ecológicas, hábitos tróficos y adaptación morfológica.

\section{Análisis general del grupo}

Al comenzar el itinerario, no se apreciaron, la gran diversidad de elementos y relaciones existentes entre las temáticas vistas y los Tricópteros. Sin embargo, a lo largo de las 
Bio - grafía. Escritos sobre la Biología y su Enseñanza. ISSN 2027-1034

\section{Edición Extraordinaria. p.p. 966 - 974}

Memorias del IX Encuentro Nacional de Experiencias en Enseñanza de la Biología y la
Educación Ambiental. IV Congreso Nacional de Investigación en Enseñanza de la
Biología.

actividades, se dio una evolución en la manera en que los estudiantes se aproximaron a las Tricópteros con relaciones complejas y sistémicas al nivel esperado para grado sexto.

En las primeras actividades los estudiantes manifestaron una aproximación a las temáticas de forma similar a la encontrada en los autores consultados (Cañal y Porlán, 1987; García y Rivero, 1993; García, 1994; Martín, 1994; Ángel y De Longhi, 2006; Brero y Prieto, 2002; Valbuena, 2007 y Martínez y Reyes, 2014) pero hacia el final del itinerario predominaron el nivel 2 y 3 de la hipótesis de progresión.

Se esperaría que con una mayor cantidad de tiempo para desarrollar los itinerarios didácticos, los porcentajes acumulados entre el nivel 2 y 3 de la hipótesis de progresión aumenten de forma considerable. No obstante, este proceso debe realizarse de forma permanente y no supeditarse a un periodo específico de tiempo.

Si se quiere tener una estimación más acertada de cómo es el aprendizaje de los estudiantes y, sobre todo, del nivel de complejidad de sus concepciones, resulta imprescindible no atenerse a una única fuente de información, dada la fuerte dependencia de las respuestas de factores como el contexto, la tarea o la dinámica previa desarrollada en el aula. También implica que cualquier afirmación que se realice debe ser considerada como una mera aproximación a una realidad compleja que no se puede reducir a relaciones de causa-efecto. Reflexiones similares fueron encontradas por Cañal y Porlán (1987); García y Rivero (1993) y García (1994) al realizar seguimiento al aprendizaje de estudiantes de secundaria utilizando como herramienta la hipótesis de progresión.

\section{CONCLUSIONES}

- La construcción de la hipótesis de progresión permitió evaluar el aprendizaje de los estudiantes en las temáticas propuestas. Por lo tanto, esta herramienta se consolidó en un insumo importante a la hora de hacer seguimiento a la manera en que los estudiantes comprendieron y relacionaron las temáticas entre sí. Además, la HDP facilitó tomar elementos propios de la práctica del profesor que permitieron proponer, ejecutar y evaluar seguimientos alternativos de enseñanza-aprendizaje de acuerdo al conocimiento deseable esperado en los estudiantes.

- La concordancia que hubo entre la secuenciación de los contenidos y las actividades desarrolladas para hacer seguimiento al aprendizaje de los estudiantes fue pertinente para esta investigación. Desde este punto de vista, la implementación de conceptos básicos relacionados con los Tricópteros fue de gran importancia para que los estudiantes dejaran la forma lineal y poco causal de pensar para tomar posturas relacionales y sistémicas.

- La mayor resistencia encontrada a la hora de hacer seguimiento al aprendizaje de los estudiantes se relacionó principalmente con la tendencia a volver a las ideas lineales y no relacionales acerca de los temas abordados. Sin embargo, tras la instrucción y el desarrollo de las actividades propuestas, los estudiantes 
Bio - grafía. Escritos sobre la Biología y su Enseñanza. ISSN 2027-1034

Edición Extraordinaria. p.p. 966 - 974

Memorias del IX Encuentro Nacional de Experiencias en Enseñanza de la Biología y la Educación Ambiental. IV Congreso Nacional de Investigación en Enseñanza de la Biología.

progresaron en la medida que tomaron sus ideas previas y las confrontaron con las nuevas, con el fin de enriquecer su conocimiento acerca de los Tricópteros.

- A lo largo de la investigación fue evidente la dificultad por parte de los estudiantes de reconocer elementos propios del microcosmos tales como los hongos, las bacterias, las algas y poblaciones de insectos. En contraste, los elementos cercanos e inmediatos a su realidad como el hábitat, el agua, las rocas y otras poblaciones de plantas y animales, fueron utilizadas en la construcción que los estudiantes realizaron desde las temáticas de ciencias naturales. Las relaciones posibles entre el microcosmos y el mesocosmos fueron el mayor obstáculo que tuvieron los estudiantes sobre todo por la dificultad para establecer cuáles eran los elementos vinculantes entre ellos.

- La acción humana fue considerada un elemento aislado de las dinámicas ecológicas de los Tricópteros con su ambiente. En la gran mayoría de actividades realizadas ésta no fue tenida en cuenta como un factor influyente en los ecosistemas acuáticos. Esto correspondió con una visión antropocéntrica de la naturaleza, en la que la acción humana es asumida como única dentro de todas las especies del planeta sin afectar a otros seres vivos. Fue necesaria la instrucción en este tema hacia el final del itinerario, para que los estudiantes comprendieran la influencia de las actividades humanas sobre los Tricópteros.

\section{BIBLIOGRAFÍA}

Ángel, G y De Longhi, A. (2006). Propuesta curricular de hipótesis de progresión para conceptos estructurantes en ecología. Cátedras de Didáctica General y Especial, Departamento de Enseñanza, Facultad de Ciencias Exactas, Físicas y Naturales, Universidad Nacional de Córdoba.

Cañal, P. (1994). Los ámbitos de investigación como organizadores del conocimiento escolar en la propuesta curricular investigando nuestro mundo. Investigación en la escuela (23), 87-94.

Cañal, P y Porlán, R. (1987). Investigando la realidad próxima: un modelo didáctico alternativo. Enseñanza de las ciencias, 5 (2), 89-96.

García, F, y Rivero, G. (1993). Dificultades y obstáculos en la construcción del conocimiento escolar en una hipótesis de progresión de lo simple a lo complejo. Reflexiones desde el ámbito del medio urbano. Departamento de didáctica de las ciencias. Facultad de ciencias de la educación. Sevilla. 
Bio - grafía. Escritos sobre la Biología y su Enseñanza. ISSN 2027-1034

Edición Extraordinaria. p.p. 966 - 974

Memorias del IX Encuentro Nacional de Experiencias en Enseñanza de la Biología y la Educación Ambiental. IV Congreso Nacional de Investigación en Enseñanza de la Biología.

García, J. (1994). El conocimiento escolar como un proceso evolutivo: aplicación al conocimiento de nociones ecológicas. Investigación en la escuela, 23, 23-76.

García, J. (1995). Epistemología de la complejidad y enseñanza de la ecología. El concepto de ecosistema en la educación secundaria. Tesis doctoral. Universidad de Sevilla.

Porlán, R; Martín Del Pozo, R; Rivero, A; Harres, J; Azcarate, P; Pizzato, M. (2010). El cambio del profesorado de ciencias I: marco teórico y formativo. Enseñanza de las ciencias, 28, 31-46.

Valbuena, E. (2007). El conocimiento didáctico del contenido biológico: estudio de las concepciones disciplinares y didácticas de futuros docentes de la Universidad Pedagógica Nacional (Colombia). Tesis doctoral. Universidad complutense de Madrid. 